Documentation et bibliothèques

\title{
Impact des restrictions budgétaires sur les bibliothèques et centres de documentation du Québec
}

\author{
Michelle Ouellette, Maryse Dumas, Micheline Gaudette et Silvana Vendrame
}

Volume 28, numéro 4, octobre-décembre 1982

URI : https://id.erudit.org/iderudit/1053654ar

DOI : https://doi.org/10.7202/1053654ar

Aller au sommaire du numéro

Éditeur(s)

Association pour l'avancement des sciences et des techniques de la

documentation (ASTED)

ISSN

0315-2340 (imprimé)

2291-8949 (numérique)

Découvrir la revue

Citer cet article

Ouellette, M., Dumas, M., Gaudette, M. \& Vendrame, S. (1982). Impact des restrictions budgétaires sur les bibliothèques et centres de documentation du Québec. Documentation et bibliothèques, 28(4), 163-166.

https://doi.org/10.7202/1053654ar

Tous droits réservés (c) Association pour l'avancement des sciences et des techniques de la documentation (ASTED), 1982
Ce document est protégé par la loi sur le droit d'auteur. L'utilisation des services d’Érudit (y compris la reproduction) est assujettie à sa politique d'utilisation que vous pouvez consulter en ligne.

https://apropos.erudit.org/fr/usagers/politique-dutilisation/ 


\section{chronique}

\section{Impact des restrictions budgétaires}

sur les bibliothèques

et centres de documentation du Québec

Le 18 juin 1982, le Comité ad hoc sur les fermetures de services documentaires remettait au Bureau de l'ASTED un rapport sur l'impact des restrictions budgétaires au sein des bibliothèques et centres de documentation. En voici les principaux éléments.

\section{Méthode de travail}

Les résultats du rapport ont été obtenus par le biais d'une enquête couvrant l'ensemble du territoire québécois et s'adressant à tous les types de bibliothèques et de centres de documentation. On procéda à partir d'un échantillonnage sélectif de six cents bibliothèques et centres de documentation par le truchement d'une lettre accompagnée d'un questionnaire.

\section{Analyse des résultats}

Le taux de réponses a été très élevé, soit $38,8 \%$ (233 sur 600). L'analyse des résultats s'est faite à partir de ces données. Sur les 233 réponses, il est à noter que $55,8 \%$ proviennent de la région de Montréal, $16,7 \%$ de celle de Québec, alors que le reste $(27,5 \%)$ est réparti dans les sept autres régions de la province. Cette très forte concentration de réponses en provenance des deux grandes régions urbaines de la province $(72,5 \%)$ reflète bien la répartition de la population sur le territoire.

La plus grande participation est celle du secteur Education: $34,3 \%$ des réponses représentent les milieux scolaire, collégial et universitaire, ainsi que les commissions scolaires. Vient ensuite le secteur Santé Centre hospitalier $(\mathrm{CH})$. Centre des services sociaux (CSS). Département de santé communautaire (DSC), etc. avec un taux de $21,9 \%$, puis le secteur Municipal (comprenant autant les bibliothèques publiques que les bibliothèques centrales de prêt $B C P$, qui regroupe $20,2 \%$ des réponses.

\section{Restrictions budgétaires}

Pour la prochaine année fiscale, $28,7 \%$ des bibliothèques subiront une baisse de leur budget alors que $27,5 \%$ conserveront un budget identique à l'année précédente, ce qui équivaut à une diminution compte tenu du taux d'inflation: $56,2 \%$ se ressentiront donc de l'effet des compressions. Seulement $32,6 \%$ des bibliothèques verront leur budget augmenter $(11,2 \%$ des bibliothèques n'ont pas répondu à cette question)

Sur les 169 bibliothèques qui ont précisé le pourcentage de l'augmentation ou de la diminution de leur budget, 11,9\% bénéficieront d'une hausse de plus de $11 \%$, alors que $13,3 \%$ n'augmenteront que de 1 à $10 \%$. En revanche, 9,5\% des bibliothèques verront leur budget réduit de 1 à $10 \%$, et encore $9,5 \%$ subiront une perte de plus de $11 \%$.

\section{Effectifs des bibliothèques}

1900 employés sont répartis entre les 219 bibliothèques ayant répondu à la question concernant les effectifs. $41,7 \%$ des bibliothèques ont à leur service de un à trois employés; $25,8 \%$ entre quatre et huit; $13,7 \%$ entre 9 et $19,12,4 \%$ ont plus de vingt employés (ce qui peut en certains cas signifier aussi plus de 100!).

$45,3 \%$ des postes inventoriés sont subalternes (secrétaires, commis, agents de bureau). Les bibliotechniciens occupent $25,5 \%$ des postes, alors que 16,2\% le sont par des bibliothécaires. Enfin, 8,1\% des postes sont occupés par des employés de formation diverse (techniciens en audiovisuel, techniciens en administration, etc.).

II est intéressant de constater que $77,5 \%$ des bibliothèques ont à leur emploi des bibliotechniciens, et que sur ce pourcentage $42,6 \%$ en ont plus de 
deux. Par comparaison, 44,2\% des bibliothèques emploient des bibliothécaires, et seulement $24,5 \%$ plus de deux.

Comme l'illustre le tableau I, la catégorie comprenant le plus d'employés à temps partiel est celle des commis, dans $27,1 \%$ des bibliothèques. Seulement $13,8 \%$ des bibliothèques emploient des bibliotechniciens à temps partiel, tandis que 10,2\% embauchent des bibliothécaires à temps partiel.

\begin{tabular}{|clc|}
\hline \multicolumn{2}{c|}{ TABLEAU I } \\
Temps plein & Catégorie & \\
& & Temps partiel \\
460 & Commis & 290 \\
90 & Secrétaires & 24 \\
439 & Bibliotechniciens & 47 \\
286 & Bibliothécaires & 24 \\
105 & Autres & 50 \\
\hline
\end{tabular}

\section{Fermeture de bibliothèques}

209 bibliothèques sur 231 ne prévoient pas de fermeture, alors que douze devront fermer, et que le sort de dix autres demeure encore inconnu (voir le tableau (I). II serait très intéressant de connaître les raisons justifiant la fermeture de quelque 10\% des bibliothèques, compte tenu de l'énorme investissement nécessité par l'implantation d'un service documentaire.

Des douze fermetures officielles, six seront définitives et six temporaires. Par temporaire, il faut entendre une possibilité de réouverture mais dans un avenir indéterminé.

Aucune fermeture n'est prévue dans les bibliothèques des secteurs scolaire, collégial et universitaire. Par contre, il y aura fermeture de deux bibliothèques municipales, d'une bibliothèque gouvernementale, de deux bibliothèques spécialisées privées, de trois bibliothèques du secteur de la santé et de trois bibliothèques de commissions scolaires. De plus, une dizaine d'autres pourraient connaître un sort identique.

\begin{tabular}{|c|c|c|c|}
\hline \multicolumn{4}{|c|}{ TABLEAU II } \\
\hline \multicolumn{4}{|c|}{ Fermeture par type de bibliothèques } \\
\hline $\begin{array}{l}\text { Type de } \\
\text { bibliothèques }\end{array}$ & Réponses & Fermeture & $\begin{array}{l}\text { En } \\
\text { Ission }\end{array}$ \\
\hline Municipale & 41 & 2 & 2 \\
\hline Centrale de prêt & 5 & 1 & 0 \\
\hline Gouvernementale & 27 & 1 & 2 \\
\hline Privée & 27 & 2 & 1 \\
\hline Santé & 50 & 3 & 3 \\
\hline Scolaire & 29 & 0 & 0 \\
\hline Collégiale & 30 & 0 & 0 \\
\hline Universitaire & 14 & 0 & 0 \\
\hline Commission scolaire & 7 & 3 & 1 \\
\hline \multirow[t]{2}{*}{ Autre } & 1 & 0 & 1 \\
\hline & 231 & 12 & 10 \\
\hline
\end{tabular}

\section{Coupures de postes}

Même si $90 \%$ des bibliothèques prévoient survivre, il y en aura cependant $38 \%$ qui subiront des coupures de postes, et $8,3 \%$ qui pourraient éventuellement en connaître, pour un total potentiel de $46,3 \%$ (voir tableau III).

\begin{tabular}{|ccc|}
\hline $\begin{array}{c}\text { Nombre de postes } \\
\text { coupés }\end{array}$ & $\begin{array}{c}\text { TABLEAU III } \\
\text { Nombre de bibliothèques } \\
\text { touchées }\end{array}$ & $\%$ \\
1 & 61 & 65,6 \\
2 & 12 & 12,9 \\
3 & 7 & 7,6 \\
$4-5$ & 9 & 9,7 \\
$6-8$ & 3 & 3,2 \\
$12-15$ & 1 & 1,0 \\
& & \\
& $\mathbf{9 3}$ & $\mathbf{1 0 0 .}$ \\
\hline
\end{tabular}

\section{Coupures de postes selon le type de bibliothèques}

En interprétant les pourcentages du tableau IV, on s'aperçoit que 19 bibliothèques du secteur scolaire devront couper globalement les postes de 22 employés; 18 bibliothèques collégiales devront en couper de 36 à 38; 17 bibliothèques du secteur de la santé perdront de 23 à 24 employés; neuf bibliothèques publiques verront partir de 23 à $27 \mathrm{em}$ ployés; deux $\mathrm{BCP}$ en perdront de treize à seize; dix employés perdront leur emploi dans huit bibliothèques spécialisées privées; de 24 à 29 employés seront mis à pied dans neuf bibliothèques gouvernementales; dans cinq bibliothèques universitaires, de huit à neuf personnes perdront leur poste et dans cinq commissions scolaires de neuf à dix employés n'auront plus d'emploi.

\begin{tabular}{|c|c|c|c|}
\hline \multicolumn{4}{|c|}{ TABLEAU IV } \\
\hline Type de bibliothèques & Oui $(\%)$ & Non $(\%)$ & $\begin{array}{c}\text { En } \\
\text { discussion } \\
(\%)\end{array}$ \\
\hline Municipale & 22,0 & 68,3 & 9,7 \\
\hline $\mathrm{BCP}$ & 40,0 & 60,0 & 0,0 \\
\hline Gouvernementale & 25,9 & 50,3 & 14,8 \\
\hline Privée & 30,8 & 61,5 & 7,7 \\
\hline Santé & 34,7 & 65,3 & 0,0 \\
\hline Scolaire & 55,2 & 34,5 & 10,3 \\
\hline Collégiale & 60,0 & 30,0 & 10,0 \\
\hline Universitaire & 28,6 & 35,7 & 35,7 \\
\hline Commission scolaire & 71,4 & 28,6 & 0,0 \\
\hline
\end{tabular}




\section{Postes abolis par catégorie d'employés}

Du tableau $\mathrm{V}$ on peut tirer les conclusions suivantes: il y a 172 personnes qui ont subi ou qui subiront des mises à pied. Elles représentent $9 \%$ du personnel inventorié, appartenant pour $4,2 \%$ au personnel subalterne, et pour $4,8 \%$ au personnel diplômé (bibliotechniciens, bibliothécaires, autres). On sait par ailleurs que $0,8 \%$ des postes ont été abolis après le départ volontaire des employés concernés, ce qui équivaut à dire que le personnel correspondant à l'autre $8,2 \%$ a été remercié à cause des coupures.

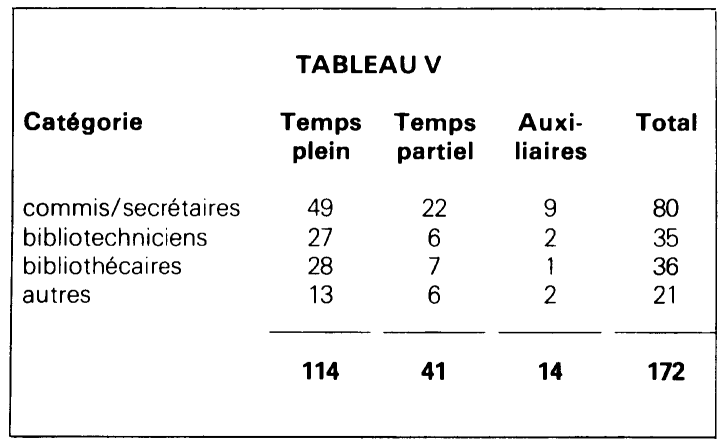

En analysant chacune de ces catégories, on remarque que $7,2 \%$ des bibliotechniciens subissent des mises à pied, que $9,2 \%$ des employés subalternes (secrétaires/commis) sont touchés, que 12,9\% des employés ayant reçu une formation diverse sont remerciés et que $11,6 \%$ des bibliothécaires vont perdre leur poste.

\section{Postes abolis par région}

$36,1 \%$ des bibliothèques de la région de Montréal (soit 47 bibliothèques) perdront un total de 87 à 97 employés. $46.1 \%$ des bibliothèques de la région de Québec (18 sur 39 ) perdront de 30 à 32 employés. Même si, de prime abord, les autres régions de la province semblent moins affectées, elles n'en subissent pas moins un très fort pourcentage de coupures de postes, ainsi que l'illustre le tableau VI.

\begin{tabular}{|lccc|}
\hline \multicolumn{3}{c}{ TABLEAU VI } & \\
Région & $\begin{array}{c}\text { Bibliothèques } \\
\text { touchées }\end{array}$ & $\%$ & $\begin{array}{c}\text { Employés } \\
\text { mis à pied }\end{array}$ \\
Abitibi & $4 / 7$ & 41,6 & 7 \\
Bas-St-Laurent & $5 / 9$ & 55,5 & 7 \\
Côte Nord & $2 / 4$ & 50,0 & $7-8$ \\
Estrie & $6 / 10$ & 60,0 & 6 \\
Mauricie & $5 / 18$ & 27,7 & $9-10$ \\
Outaouais & $2 / 7$ & 28,5 & $12-15$ \\
Saguenay & $4 / 9$ & 77,7 & 8 \\
Montréal & $47 / 130$ & 36,1 & $87-97$ \\
Québec & $18 / 39$ & 46,1 & $30-32$ \\
& & & \\
\hline
\end{tabular}

\section{Fermeture des bibliothèques et coupures selon le nombre d'années d'existence}

Le tableau VII démontre que la majorité des bibliothèques subissant des coupures de postes existent depuis dix à quinze ans $(42,5 \%) ; 20,6 \%$ existent depuis 16 à 24 ans, 17,2\% depuis plus de 25 ans, et seulement $19,5 \%$ depuis moins de neuf ans. Le plus faible pourcentage de mises à pied est obtenu par les bibliothèques ayant moins de six ans d'existence $(8 \%)$. Est-ce pur hasard, ou cela reflètet-il la fermeté d'administrateurs qui viennent de faire un investissement? Ou encore, cela signifie-t-il une meilleure planification du personnel, ou une prise de conscience de la nécessité de maintenir un service documentaire adéquat? Cela pourrait tout aussi bien étayer le fait que personne ne peut couper des postes là où il y en a déjà trop peu!

\begin{tabular}{|c|c|c|c|}
\hline \multicolumn{4}{|c|}{ TABLEAU VII } \\
\hline Années & $\begin{array}{l}\text { Bibliothèques } \\
\text { touchées }(\%)\end{array}$ & Fermeture & $\begin{array}{c}\text { Coupures } \\
(\%)\end{array}$ \\
\hline $1-9$ & 30,5 & 1 & 27,5 \\
\hline $10-15$ & 31,4 & 4 & 42,5 \\
\hline 16-18) & & 1 & \\
\hline $19-21\}$ & 27,0 & 1 & 20,6 \\
\hline $22-24)$ & & 1 & \\
\hline $25+$ & 22,3 & 3 & 17,2 \\
\hline $\begin{array}{c}\text { (réponse } \\
\text { incomplète) }\end{array}$ & & 1 & \\
\hline
\end{tabular}

Les douze bibliothèques qui fermeront leurs portes mettront de 69 à 82 employés à pied, soit de $3,6 \%$ à $4,3 \%$ du nombre total d'employés répertoriés et de $40,1 \%$ à $47,6 \%$ du nombre total d'employés mis à pied.

\section{Personnel mis à pied}

Le tableau VIII fait ressortir le fait que les employés mis à pied sont majoritairement des femmes. On peut même supposer que la majorité des 73 bibliothèques n'ayant pas répondu à cette question, emploie un personnel surtout féminin, les femmes représentant la plus grande proportion des membres de nos professions.

\begin{tabular}{|c|c|c|c|}
\hline \multirow[b]{2}{*}{ Catégorie } & \multicolumn{2}{|c|}{ TABLEAU VIII } & \multirow[b]{2}{*}{$\begin{array}{c}\text { Sans } \\
\text { réponse }\end{array}$} \\
\hline & Hommes & Femmes & \\
\hline Bibliothécaires & 9 & 17 & 10 \\
\hline Bibliotechniciens & 1 & 17 & 17 \\
\hline Personnel subalterne & 2 & 37 & 41 \\
\hline Autres & 8 & 10 & 3 \\
\hline
\end{tabular}

$34,2 \%$ des bibliotechniciens mis à pied ont entre vingt et trente ans, alors que $11,2 \%$ sont âgés de 
trente à quarante ans. II y a plus de $48,5 \%$ des bibliotechniciens pour lesquels le groupe d'âge n'a pas été précisé.

$19,4 \%$ des bibliothécaires mis à pied se retrouvent dans la catégorie des trente à quarante ans. On retrouve une proportion à peine inférieure dans chacun des autres groupes d'âge. Le taux des bibliothèques ayant ignoré cette question a atteint $36,3 \%$.

\section{Conclusions}

Aussi intéressants soient-ils, les résultats de l'enquête doivent toutefois être lus comme une série d'indications, fiables mais non absolues, sur la situation du monde de la documentation au Québec.

Parmi les facteurs venant pondérer les résultats, citons d'abord la quantité des réponses. II n'existe aucun recensement complet du nombre de bibliothèques et de centres documentaires québécois. Au moment de l'envoi du questionnaire, un échantillonnage sélectif de six cents bibliothèques semblait très valable. Toutefois, la proportion réelle de ces bibliothèques par rapport au nombre total des institutions est sans doute beaucoup moins élevée que celle indiquée par le taux de réponses.

De plus, il ne faut pas oublier que les bibliothèques et centres de documentation déjà fermés n'ont bien évidemment pas été rejoints par l'enquête. Leur nombre, le sort de leur personnel et de leurs collections demeurent les grandes inconnues de la situation actuelle. Soulignons aussi le très grand nombre d'institutions sollicitées qui n'ont pas répondu à l'appel. Leur situation est-elle pour autant différente? Si oui, dans quel sens et dans quelles proportions?

Malgré tout, les indications recueillies sont précieuses, puisqu'uniques, et fiables. II suffit de reconnaître à travers les chiffres et les pourcentages la vision de faits bien réels pour être impressionné par la cohérence de l'ensemble.

\section{Situation de l'emploi}

En ce qui concerne l'avenir du personnel oeuvrant dans le domaine de la documentation, les commentaires à faire abondent. D'abord, avec un taux canadien de chômage de $10 \%$ et un taux québécois encore supérieur, on peut se demander si $9 \%$ de mises à pied ne correspond pas à une certaine norme... anormale et indésirable, mais inévitable.

En soustrayant du $9 \%$ initial un $0,8 \%$ correspondant aux postes abolis ou laissés vacants à la suite de départs volontaires, puis en l'amputant d'un autre $4,2 \%$ (personnel subalterne), il reste à peine $4 \%$ du personnel affecté pour les deux groupes spécialisés que sont les techniciens en documentation et les bibliothécaires. Malheureusement, il faut bien se rappeler qu'il s'agit de coupures de postes. A ces pourcentages, il faut donc ajouter tous les diplômés qui n'occupent pas encore un emploi régulier et tous ceux à la recherche d'un poste, quel qu'il soit.

Quant au personnel en poste, rappelons que $46,3 \%$ des réponses révèle qu'il a été ou pourra être touché par des coupures de postes. Une conclusion s'impose donc: la charge de travail va augmenter. En corollaire, ajoutons que la qualité du travail sera peut-être elle aussi atteinte dans bon nombre de cas, puisque l'équipe locale variait entre une et huit personnes pour $67,5 \%$ des bibliothèques. Toutes proportions gardées, le bibliotechnicien semble plus susceptible d'être affecté par ce phénomène que le bibliothécaire. D'abord, parce que les bibliotechniciens sont plus nombreux dans les bibliothèques que les bibliothécaires. Ensuite, parce qu'il se verra plus souvent confier des tâches de commis, c'est-àdire du groupe le plus lourdement frappé par les coupures. Enfin, parce qu'il devra parfois accomplir des tâches autrement réservées aux bibliothécaires. Du côté des bibliothécaires, les changements peuvent mener à un certain retour vers des tâches identifiées comme appartenant au bibliotechnicien, surtout si ce dernier doit suppléer un commis.

\section{Budgets}

Enfin, comment passer sous silence «le nerf de la guerre», c'est-à-dire le budget? Les compressions budgétaires ont des effets sérieux sur des points importants, bien que souvent peu apparents: postes à temps complet convertis en postes à temps partiel; personnes absentes pour une période prolongée et non remplacées; diminution des achats de volumes et du nombre d'abonnements aux périodiques; réduction du personnel; heures d'ouverture écourtées dans certains cas.

A long terme, voilà autant de risques qui s'accumulent dans la balance du jugement porté par la clientèle sur la qualité du service. Toute la question de la rentabilité, avec ses exigences, refait surface.

\section{Michelle Ouellette}

Bibliothèque municipale de Montréal

\section{Maryse Dumas}

Centre de documentation

Ordre des infirmière et infirmiers du Québec

Montréal

\section{Micheline Gaudette}

Bibliothèque

Centre des services sociaux du

Montréal métropolitain

\section{Silvana Vendrame}

\section{Bibliothèque}

Centre de recherche et de statistiques sur le marché du travail

Ministère du Travail, de la main d'oeuvre et de la sécurité du revenu

Montréal

Membres du Comité ad hoc de l'ASTED sur les fermetures de services documentaires 